\title{
Sampling a Survival and Conditional Class of Archimedean Processes
}

\author{
Barro Diakarya ${ }^{1}$
}

\author{
${ }^{1}$ UFR-SEG, Université Ouaga 2, Burkina Faso
}

Correspondence: Barro Diakarya, UFR-SEG, Université Ouaga 2, Burkina Faso. Tel: 226-7860-1086. E-mail: dbarro2@gmail.com

Received: December 15, 2012 Accepted: December 26, 2012 Online Published: January 11, 2013

doi:10.5539/jmr.v5n1p53 URL: http://dx.doi.org/10.5539/jmr.v5n1p53

\begin{abstract}
Archimedean copulas form a prominent class of copulas which lead to the construction of multivariate distributions involving one-dimensional generator functions. This paper investigate properties of Archimedean copulas of stochastic processes. Specifically we propose analytical expressions of the survival copulas of Archimedean processes. The parametric survival distributions of Archimedean copulas are also characterized. We give conditional characterizations for Archimax copulas both for strictly Archimedean and for strictly extremal subclasses in parametric context.
\end{abstract}

Keywords: copula, Archimedean, generator, survival, Archimax, extreme values

2010 Mathematical Subject Classification: 60G09, 60G70, 62N99

\section{Introduction}

In multivariate stochastic analysis the use of copulas offers a convenient framework for modelling and capturing the dependence of two or more random variables. The term "copula" was first introduced by Abe Sklar (1959) while modelling a structure of stochastic dependence for given marginal laws. Thenceforth, copulas have been largely used to construct multivariate distributions even when the univariate margins are not identically distributed (Nelsen, 2007). For example in risk management, probabilistic statements about portfolio returns would be misleading unless one has a realistic model of the multivariate distribution of the assets returns. By the theorem of Sklar (1959), copulas appear implicitly in any multivariate distribution as linked functions that join the univariate marginal to form joint distribution functions. Specifically a copula $C$ associated to a random vector $\left(X_{1}, \ldots, X_{n}\right)$ with continuous margins $\left\{F_{i} ; i=1, . ., n\right\}$ is defined on the unit cube $[0,1]^{n}$ as the joint distribution function of the uniform vector $\left(U_{1}, \ldots, U_{n}\right)$ obtained via the probability integral transformations $U_{i}=F_{i}\left(X_{i}\right)$, that is, for all $\left(u_{1}, \ldots, u_{n}\right) \in[0,1]^{n}$,

$$
\left.C\left(u_{1}, \ldots, u_{n}\right)=P\left[F_{1}\left(X_{1}\right) \leq u_{1}, \ldots, F_{n}\left(X_{n}\right) \leq u_{n}\right)\right] .
$$

Standard references for copulas analysis are Joe (1997) or Nelsen (2007) which provides detailed and readable introductions to copulas and their statistical and mathematical foundations. Other Probabilistic and statistical aspects of copulas are to be found for instance in McNeil et al. (2009) or in Müller et al. (2005) while Embrechts et al. (2001) perform copula-based algorithms for numerical simulations for risk management purposes.

Archimedean copulas not only arise naturally in the context of Laplace Transform (Joe, 1997), they form also an important family that allows multivariate dependence modelling involving one-dimensional generator functions (Charpentier et al., 2009). Following McNeil (2008), a n-dimensional copula $\mathrm{C}$ is a Archimedean copula if, for all $\left(u_{1}, \ldots, u_{n}\right) \in[0,1]^{n}$, it has the simple algebraic representation

$$
C_{\varphi}\left(u_{1}, \ldots, u_{n}\right)=\varphi\left[\varphi^{-1}\left(u_{1}\right)+\ldots+\varphi^{-1}\left(u_{n}\right)\right],
$$

where $\varphi$ is a specific function called Archimedean generator of $C_{\varphi}$. Archimedean copulas present particular attracted interest due to their analytical form given by the generator and which make them simple to analysis and their exchangeability. The particular case of bivariate Archimedean copulas have been largely developped by Genest and Mackay (1986) while tractable Archimedean families are applied in multivariate survival analysis by Clayton (1978) and in actuariat loss by Klugman et al. (1999). 
In quantitative finance field however portfolio managers need non-exchangeable models of copulas (Bouyé, 2002). More generally, in situation where causality among stochastic process flows in certain direction, symmetry property turns out to be restrictive. That prompted Capérà et al. (2000) to model in bivariate context a new family called Archimax copulas which combines both Archimedean and extreme values models.

The paper is organized as follows: Section 2 collects preliminary tools of multivariate analysis that turn out to be necessary for our results. Section 3 presents our main results. Specifically, an analogue to the Sklar theorem is discovered but for a parametric family of Archimedean copulas and in a survival context. Then it is proved that the copula associated to the survival distribution of Archimedean copula is still Archimedean. Finally, Section 4 deals with sampling conditional copulas both for parametric Archimedean and for Archimax subfamilies.

\section{Materials and Methods}

In this section we collect important definitions and usefull properties on the copula associated to a conditional distribution function and the copulas of Archimedean and Archimax subfamilies. These results turn out to be necessary for our approach. We refer the reader to Genest and Mackay (1986) for bivariate Archimedean copulas and to Cherubini et al. (2004) for Archimedean copulas applications in different degrees of quantitative finance.

\subsection{An Overview on Copulas of Conditional Distributions}

A complete definition of multivariate copulas will be needed for further discussions.

Definition 1 A n-dimensional copula is a multivariate distribution function $C:[0,1]^{n} \longrightarrow[0,1]$ satisfying the following properties

i) $C\left(u_{1}, \ldots, u_{i-1}, 0, u_{i+1}, \ldots, u_{n}\right)=0$ for all $\left(u_{1}, \ldots, u_{i-1}, u_{i+1}, \ldots, u_{n}\right) \in[0,1]^{n-1}$.

ii) $C\left(1, \ldots, 1, u_{i}, 1, \ldots, 1\right)=u_{i}$ for all $u_{i} \in[0,1], i=1,2, \ldots, n$.

iii) $\mathrm{C}$ is n-increasing, i.e., the volume $V_{B}$ of any hyperrectangle $B=\prod_{i=1}^{n}\left[a_{i}, b_{i}\right] \subseteq[0,1]^{n}$ is positive,

$$
V_{B}=\int_{B} d C\left(u_{1}, \ldots, u_{n}\right)=\sum_{i_{1}=1}^{2} \ldots \sum_{i_{n}=1}^{2}(-1)^{i_{1}+\ldots+i_{n}} C\left(u_{i_{1}}, \ldots, u_{i_{n}}\right) \geq 0 .
$$

While modelling dependence of asymmetric time-varying exchange rate, Patton (2006) pointed out the importance of conditional copulas in financial market analysis. Let $X_{1}$ and $X_{2}$ be two random variables with joint distribution $F=\left(F_{1}, F_{2}\right)$ and $\mathrm{W}$ be a conditioning set in $\mathbb{R}^{2}$.

Theorem 2 (Patton, 2006) Let $\left\{F_{i, W} ; i=1,2\right\}$ be the conditional distributions of $X_{i} \mid W$ and $F_{W}$ the joint conditional distribution of $\left(X_{1}, X_{2}\right) / W$. Assume that $F_{i, W}$ are continuous on $\overline{\mathbb{R}}$. Then there exists a unique conditional copula $C_{W}$ such as, for all $\left(x_{1}, x_{2}\right) \in(\overline{\mathbb{R}})^{2}$,

$$
F_{W}\left(x_{1}, x_{2} \mid w\right)=C_{W}\left(F_{1, W}\left(x_{1} \mid w\right), F_{2, W}\left(x_{2} \mid w\right) \mid w\right) ; w \in W .
$$

Conversely, if we let $F_{i, W}$ be the conditional distribution of $X_{i} \mid W$ and $C_{W}$ be a conditional copula, then the function $F_{W}$ defined by (4) is a conditional bivariate distribution function with conditional marginal distribution $F_{i, W}$.

Note that Theorem 2 provides the conditional version of the theorem of Sklar (1959). It means in particular that the conditional copula $\mathrm{C}_{W}$ of $\left(X_{1}, X_{2}\right) / W$ is the joint distribution function of $\left(U_{1}, U_{2}\right) \sim\left(F_{1}\left(X_{1} / W\right), F_{2}\left(X_{2} / W\right)\right)$ given $W$.

\subsection{An Overview on Archimedean and Archimax Copulas}

The concept of Archimedean copula is inherently related to Archimedean generator.

Definition 3 (Archimedean generator) A continuous and strictly decreasing function $\varphi:[0,+\infty] \longrightarrow[0,1]$ is called Archimedean generator if it is convex and satisfies the condition $\varphi(0)=1$ and $\varphi(+\infty)=0$.

A Archimedean copula satisfies the relation (2) for some generator $\varphi$ with inverse $\varphi^{-1}:[0,1] \longrightarrow[0,+\infty]$ such as, for all $\left(u_{1}, \ldots, u_{n}\right) \in[0,1]^{n}$;

$$
C\left(u_{1}, \ldots, u_{n}\right)=\varphi\left[\varphi^{-1}\left(u_{1}\right)+\ldots+\varphi^{-1}\left(u_{n}\right)\right] \text { with } \varphi^{-1}(y)=\inf \{t \in[0,1]: \varphi(t) \leq y\} .
$$

McNeil et al. (2009) show that a necessary and sufficient condition for the right hand side of (2) to be a copula is 
that $\varphi^{-1}$ is completely monotone on $[0 ; \infty]$, that is, it is $(n-2)$ times derivative and

$$
(-1)^{k}\left(\varphi^{-1}\right)^{(k)}(t) \geq 0 \text { for all } k=1,2, \ldots, n-2,
$$

and $(-1)^{k}\left(\varphi^{-1}\right)^{(n-2)}$ is non-negative, non-increasing and convex on $[0 ; \infty]$.

The class of Archimax copulas contains a special subclass of all Archimedean copulas and all extreme values copulas (see Capéràa et al., 2000). A bivariate member of this class with generator $\varphi$ is in the form

$$
C_{\varphi, A}(u, v)=\varphi^{-1}\left(\min \left[\varphi(0), \varphi(u)+\left(\varphi(v) A\left(\frac{\varphi(u)}{\varphi(u)+\varphi(v)}\right)\right]\right) ;(u, v) \in[0,1]^{2} ;\right.
$$

where, in multivariate case, A is a Pickands (1981) dependence function mapping the unit simplex $S_{n}$ of $\mathbb{R}^{n}$, given by

$$
S_{n}=\left\{x \in \mathbb{R}^{n} ;\|x\|_{1}=1 ;\|x\|_{1}=\sum_{i=1}^{n} x_{i}\right\},
$$

to $\left[\frac{1}{n}, 1\right]$, satisfying $\max (t ; 1-t) \leq A(t) \leq 1$ for all $t \in[0 ; 1]$.

\section{Analytical Form of Survival Copulas of Archimedean Processes}

Let $\left\{X_{t}=\left(X_{t, 1}, \ldots, X_{t, n}\right), t \in \chi\right\}$ be a continuous stochastic random vector with distribution $F_{t}=\left(F_{t, 1}, \ldots, F_{t, n}\right)$ where $\chi$ is a set (time or space) of parameters, see (Schmitz, 2003). The following definition introduces the concept of Archimedean Process.

Definition 4 Let $\left\{\varphi_{t}, t \in \chi\right\}$ be a parametric Archimedean generator. A continuous stochastic process $\left\{X_{t}, t \in \chi\right\}$ is called Archimedean process with generator $\varphi_{t}$ (or a $\varphi_{t}$-Archimedean process) if its corresponding copula is the Archimedean copula $C_{\varphi_{t}}$, that is, generated by $\varphi_{t}$.

Particularly, if the process $\left\{X_{t}, t \in \chi\right\}$ deals with reliability theory in actuarial science, duration analysis in economics or event history analysis in sociology, it is more appropriate to use the complementary distribution function or survival distribution $\bar{F}_{t}$ given for $\left(x_{t, 1}, \ldots, x_{t, n}\right) \in \overline{\mathbb{R}}^{n}, t \in \chi$ by

$$
\bar{F}_{t}\left(x_{t, 1} ; \ldots ; x_{t, n}\right)=P\left[X_{t, 1}>x_{t, 1} ; \ldots ; X_{t, n}>x_{t, n}\right] .
$$

The following key result provides the analytical form for survival copulas of a Archimedean process.

Theorem 5 Let $\bar{C}_{\varphi_{t}}$ be the survival copulas of a $\varphi_{t}$-Archimedean process $\left\{X_{t}, t \in \chi\right\}$. Then, for all $\left(u_{t, 1 ; \cdots} ; u_{t, n}\right) \in$ $[0,1]^{n}$,

$$
\bar{C}_{\varphi_{t}}\left(u_{t, 1} ; \ldots ; u_{t, n}\right)=\sum_{k=0}^{n}\left[(-1)^{k}\left(\sum_{S_{k} \subset N}\left[\varphi_{t}\left(\sum_{j=1}^{n} \varphi_{t}^{-1}\left(1-u_{t, i_{j}}\right)\right)\right]\right)\right]
$$

for specific $k$-dimensional elements $S_{k}=\left\{i_{1}, i_{2}, \ldots, i_{k}\right\}$ of the set of non-empty subsets of $N=\{1,2, \ldots, n\}$.
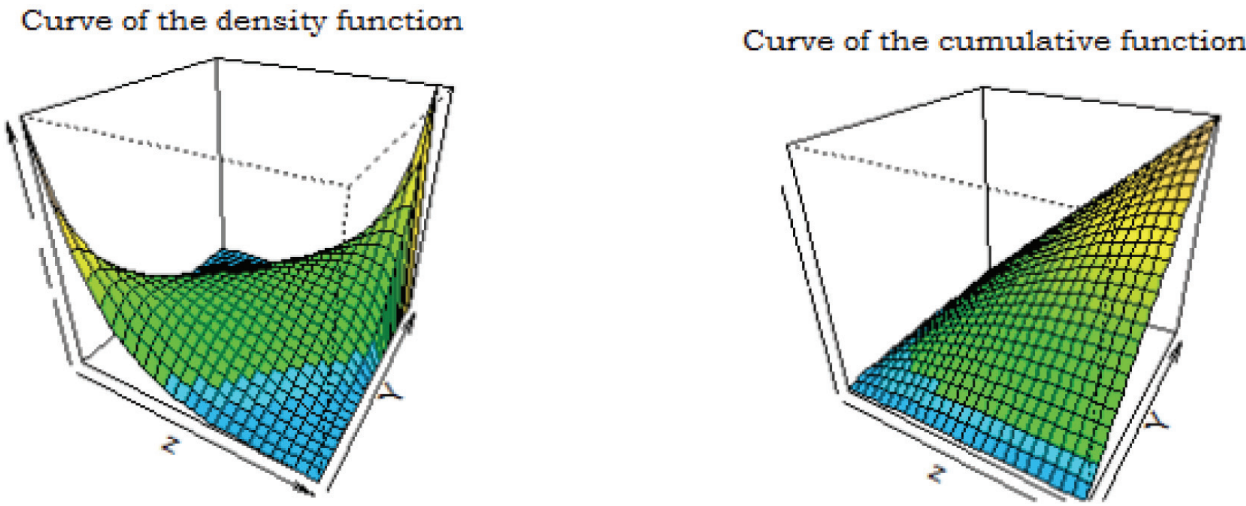

An example of Graphic of Parametric bivariate Archimedean

Figure 1. Graphic of a bivariate Archimedean copula 
Proof. Definition 4 means that, for all $t \in \chi$, the copula $C_{\varphi_{t}}$ of a $\varphi_{t}$-Archimedean process has the form

$$
C_{\varphi_{t}}\left(u_{1}, \ldots, u_{n}\right)=\varphi_{t}\left[\varphi_{t}^{-1}\left(u_{t, 1}\right)+\ldots+\varphi_{t}^{-1}\left(u_{t, n}\right)\right] ;\left(u_{t, 1} ; \ldots ; u_{t, n}\right) \in[0,1]^{n} .
$$

Moreover, since any copula is also a cumulative distribution, then by denoting $\tilde{C}$ the survival distribution of the survival copula $\bar{C}$, it follows from Frahm (2006) that, for all $\left(u_{1}, \ldots, u_{n}\right) \in[0,1]^{n}$,

$$
\bar{C}\left(u_{1}, \ldots, u_{n}\right)=\tilde{C}\left(1-u_{1}, \ldots, 1-u_{n}\right) .
$$

Then, using the Sylvester-Poincaré sieve formula (see Durante et al., 2010), we obtain that;

$$
\tilde{C}\left(u_{1}, \ldots, u_{n}\right)=\sum_{k=0}^{n}\left[(-1)^{k}\left(\sum_{v\left(u_{1}, \ldots, u_{n}\right) \in Z(n-k, n, 1)} C\left(v_{1}, \ldots, v_{n}\right)\right)\right],
$$

where $Z(M, N, \varepsilon)$ denotes the set $\left\{v \in[0,1]^{n} ; v_{i} \in\left\{u_{i}, \varepsilon\right\}, \sum_{i=1}^{n} 1_{\{\varepsilon\}}\left(v_{i}\right)=M\right\}$.

Then, we check easily that the formula (11) can be written more simply as, for all $\left(u_{1}, \ldots u_{n}\right) \in[0,1]^{n}$,

$$
\left.\tilde{C}\left(u_{1}, \ldots, u_{n}\right)=\sum_{k=0}^{n}\left[(-1)^{k} \sum_{S_{k} \subset N} C\left(u_{i_{1}} ; \ldots ; u_{i_{n}}\right) ; i_{j} \in S_{k}\right)\right] ;
$$

where $S_{k}=\left\{i_{1}, i_{2}, \ldots, i_{k}\right\}$ are k-dimensional elements of the set of non-empty subsets of $N=\{1,2, \ldots, n\}$ with where $u_{i_{j}}=1$ for all $i_{j} \notin S_{k}$. Therefore, by replacing (12) in (10) it follows that, for all $\left(u_{1}, \ldots, u_{n}\right) \in[0,1]^{n}$,

$$
\bar{C}\left(u_{1}, \ldots, u_{n}\right)=\sum_{k=0}^{n}\left[(-1)^{k}\left(\sum_{S_{k} \subset N} C\left(1-u_{i_{1}} ; \ldots ; 1-u_{i_{n}}\right)\right)\right],
$$

where $S_{k}$ is rather such as $u_{i_{j}}=0$ for all $i_{j} \notin S_{k}$. Particularly, for a parametric $\varphi_{t}$-Archimedean process $\left(X_{t}, t \in \chi\right)$, it follows from (13) that,

$$
\bar{C}_{\varphi_{t}}\left(u_{t, 1} ; \ldots ; u_{t, n}\right)=\sum_{k=0}^{n}\left[(-1)^{k} \sum_{S_{k} \subset N} C_{\varphi_{t}}\left(1-u_{t, i_{1}} ; \ldots ; 1-u_{t, i_{n}}\right)\right] \text { with } u_{t, i_{k}}=0, i_{k} \notin S_{k}
$$

Finally, by replacing $C_{\varphi_{t}}$ by its analytical expression (9) in (14) the result (8) follows as disserted.

Theorem 6 Let $\bar{F}_{t}$ be the n-dimensional survival function of a $\varphi_{t}$-Archimedean process. Then there exists a parametric copula $\bar{C}_{\varphi_{t}}$ satisfying (1), that is, for all $\left(u_{t, 1} ; \ldots ; u_{t, n}\right) \in[0,1]^{n}, t \in \chi$.

$$
\left.\bar{C}_{\varphi_{t}}\left(u_{t, 1} ; \ldots ; u_{t, n}\right)=P\left[\bar{F}_{t, 1}\left(X_{t, 1}\right) \leq u_{t, 1} ; \ldots ; \bar{F}_{t, n}\left(X_{t, n}\right) \leq u_{t, n}\right)\right] ;
$$

where $\bar{F}_{t, i} ; i=1, \ldots, n$ are the survival margins of the process. Moreover, $\bar{C}_{\varphi_{t}}$ is the survival copula of the process.

Proof. In copulas analysis, multivariate properties result from symmetric or asymmetric extensions of bivariate case. So, there is no loss of generality by proving Theorem 6 for 2 or 3-dimensional case. For this end, consider the copulas $C_{\varphi_{t}}$ of a 3 -dimensional $\varphi_{t}$-Archimedean process.

Then, from the formula (13) and for a specific $S_{k}$, it follows that, for all $u_{t, i} \in[0,1] ; i=1,2,3$;

$$
\bar{C}_{\varphi_{t}}\left(u_{t, 1} ; u_{t, 2} ; u_{t, 3}\right)=\sum_{k=0}^{3}\left[(-1)^{k}\left(\sum_{S_{k} \subset\{1,2,3\}} C_{\varphi_{t}}\left(1-u_{t, 1_{k}} ; \ldots ; 1-u_{t, i_{k}}\right)\right)\right] ; t \in \chi .
$$

Then, after some algebra, we obtain

$$
\begin{aligned}
\tilde{C}_{\varphi_{t}}\left(u_{t, 1} ; u_{t, 2} ; u_{t, 3}\right) & =C_{\varphi_{t}}(1,1,1)-C_{\varphi_{t}}\left(u_{t, 1} ; 1 ; 1\right)-C_{\varphi_{t}}\left(1 ; u_{t, 2} ; 1\right)-C_{\varphi_{t}}\left(1 ; 1 ; u_{t, 3}\right) \\
& +C_{\varphi_{t}}\left(1 ; u_{t, 2} ; u_{t, 3}\right)+C_{\varphi_{t}}\left(u_{t, 1} ; 1 ; u_{t, 3}\right)+C_{\varphi_{t}}\left(u_{t, 1} ; u_{t, 2} ; 1\right)-C_{\varphi_{t}}\left(u_{t, 1} ; u_{t, 2} ; u_{t, 3}\right) .
\end{aligned}
$$

By denoting $C_{i, j, \varphi_{t}}$ the marginal copulas of $C_{\varphi_{t}}$ related to $\{i, j\}$ the relation (17) yields,

$$
\begin{aligned}
\tilde{C}_{\varphi_{t}}\left(u_{t, 1} ; u_{t, 2} ; u_{t, 3}\right)= & 1-u_{t, 1}-u_{t, 2}-u_{t, 3}+C_{2,3, \varphi_{t}}\left(u_{t, 2} ; u_{t, 3}\right)+C_{1,3, \varphi_{t}}\left(u_{t, 1} ; u_{t, 3}\right) \\
& +C_{1,2, \varphi_{t}}\left(u_{t, 1} ; u_{t, 2}\right)-C_{\varphi_{t}}\left(u_{t, 1} ; u_{t, 2}, u_{t, 3}\right) .
\end{aligned}
$$


Then, applying the relation (10) the survival copula $\bar{C}_{\varphi_{t}}$ is given, for all $u_{t, i} \in[0,1] ; t \in \chi$, by

$$
\left.\begin{array}{c}
\bar{C}_{\varphi_{t}}\left(u_{t, 1}, u_{t, 2}, u_{t, 3}\right)=u_{t, 1}+u_{t, 2}+u_{t, 3}-2+C_{2,3, \varphi_{t}}\left(1-u_{t, 2}, 1-u_{t, 3}\right)+C_{1,3, \varphi_{t}}\left(1-u_{t, 1}, 1-u_{t, 3}\right) \\
+C_{1,2 \varphi_{t}}\left(1-u_{t, 1}, 1-u_{t, 2}\right)-C_{\varphi_{t}}\left(1-u_{t, 1}, 1-u_{t, 2}, 1-u_{t, 3}\right)
\end{array}\right\}
$$

Furthermore, Clayton (1978) showed that a lower dimensional marginal of a multivariate Archimedean copula is still an Archimedean copula with the same generator. So, the relation (18) yields

$$
\left.\begin{array}{rl}
\bar{C}_{\varphi_{t}}\left(u_{t, 1}, u_{t, 2}, u_{t, 3}\right)= & u_{t, 1}+u_{t, 2}+u_{t, 3}-2+\varphi_{t}\left[\varphi_{t}^{-1}\left(1-u_{t, 2}\right)+\varphi_{t}^{-1}\left(1-u_{t, 3}\right)\right] \\
& +\varphi_{t}\left[\varphi_{t}^{-1}\left(1-u_{t, 1}\right)+\varphi_{t}^{-1}\left(1-u_{t, 3}\right)\right]+\varphi_{t}\left[\varphi_{t}^{-1}\left(1-u_{t, 1}\right)+\varphi_{t}^{-1}\left(1-u_{t, 2}\right)\right] \\
& -\varphi_{t}\left[\varphi_{t}^{-1}\left(1-u_{t, 1}\right)+\varphi_{t}^{-1}\left(1-u_{t, 2}\right)+\varphi_{t}^{-1}\left(1-u_{t, 3}\right)\right] .
\end{array}\right\}
$$

It is sufficient to prove that $\bar{C}_{\varphi_{t}}$ is a trivariate copula, that is, it satisfies properties of Definition 1. For this end, let's remark that the definition of $\varphi_{t}$ and the relation (5) ensure the bijectivity of $\varphi_{t}$. So, for all $x$ in $\overline{\mathbb{R}} ; \varphi_{t} \circ \varphi_{t}^{-1}(x)=x$ while $\varphi_{t}(\infty)=0=\varphi_{t}^{-1}(1)$.

i) For all $\left(u_{t, 1} ; u_{t, 2} ; u_{t, 3}\right) \in[0,1]^{3} ; t \in \chi$

$$
\begin{aligned}
\bar{C}_{\varphi_{t}}\left(0 ; u_{t, 2} ; u_{t, 3}\right)= & u_{t, 2}+u_{t, 3}-2+\varphi_{t}\left[\varphi_{t}^{-1}\left(1-u_{t, 2}\right)+\varphi_{t}^{-1}\left(1-u_{t, 3}\right)\right]+\varphi_{t}\left[\varphi_{t}^{-1}\left(1-u_{t, 3}\right)\right] \\
& +\varphi_{t}\left[\varphi_{t}^{-1}\left(1-u_{t, 2}\right)\right]-\varphi_{t}\left[\varphi_{t}^{-1}\left(1-u_{t, 2}\right)+\varphi_{t}^{-1}\left(1-u_{t, 3}\right)\right]=0 .
\end{aligned}
$$

Then, the exchangeability yieds that $\left.\left.\bar{C}_{\varphi_{t}}\left(0 ; u_{t, 2} ; u_{t, 3}\right)\right)=\bar{C}_{\varphi_{t}}\left(u_{t, 1} ; 0 ; u_{t, 3}\right)\right)=\bar{C}_{\varphi_{t}}\left(u_{t, 1} ; u_{t, 2} ; 0\right)=0$.

ii) For all $u_{t, i} \in[0,1]$ we check easily that $\left.\bar{C}_{\varphi_{t}}\left(1,1, u_{t, 3}\right)\right)=u_{t, 3}+3 \varphi_{t}(\infty)=u_{t, 3}$ and more generally

$$
\bar{C}_{\varphi_{t}}\left(u_{t, 1} ; u_{t, 2} ; u_{t, 3}\right)=u_{t, i} \text { if } u_{t, j}=1 \text { for } 1 \leq i \neq j \leq 3 .
$$

iii) Furthermore, note that in bivariate case, the relations (3) and (9) imply respectively

$$
C\left(u_{1}, v_{1}\right)-C\left(u_{1}, v_{2}\right)-C\left(v_{1}, u_{2}\right)+C\left(u_{2}, v_{2}\right) \geq 0 \text { for } u_{i}, v_{i} \in[0,1] \text { with } u_{1} \leq v_{1}, u_{2} \leq v_{2} .
$$

and

$$
\bar{C}_{\varphi_{t}}\left(u_{t, 1} ; u_{t, 2}\right)=u_{t, 1}+u_{t, 2}-1+\varphi_{t}\left[\varphi_{t}^{-1}\left(1-u_{t, 1}\right)+\varphi_{t}^{-1}\left(1-u_{t, 2}\right)\right] .
$$

Otherwise, for all $t \in \chi$ the function $\varphi_{t}$ and $\varphi_{t}^{-1}$ are strictly decreasing and increasing respectively.

So, for all $\left(u_{t, 1} ; u_{t, 2}\right) \in[0,1],\left(v_{t, 1} ; v_{t, 2}\right) \in[0,1]$ with $u_{t, 1} \leq v_{t, 1}$ and $u_{t, 2} \leq v_{t, 2}$, it follows that,

$$
u_{t, 2}-v_{t, 2}-\varphi_{t}\left[\varphi_{t}^{-1}\left(1-u_{t, 1}\right)+\varphi_{t}^{-1}\left(1-u_{t, 2}\right)\right]+\varphi_{t}\left[\varphi_{t}^{-1}\left(1-u_{t, 1}\right)+\varphi_{t}^{-1}\left(1-v_{t, 2}\right)\right] \geq 0 .
$$

and

$$
u_{t, 1}-v_{t, 1}-\varphi_{t}\left[\varphi_{t}^{-1}\left(1-v_{t, 1}\right)+\varphi_{t}^{-1}\left(1-v_{t, 2}\right)\right]+\varphi_{t}\left[\varphi_{t}^{-1}\left(1-v_{t, 1}\right)+\varphi_{t}^{-1}\left(1-u_{t, 2}\right)\right] \geq 0 .
$$

Then, by adding (21) and (22) member to member, we obtain the $\bar{C}_{\varphi_{t}}$-version of the (20). Hence, in the bivariate survival copula $\bar{C}_{\varphi_{t}}$ is 2 -increasing and a symmetric extension allows to conclude for high dimensional case.

\section{Sampling Conditional Copulas of Archimedean Processes}

Archimax copulas have the advantage to include both the Archimedean and the strictly extremal copulas. Specifically every extremal copula $C$ is associated to a Pickands (1981) $A_{C}$ defined on $S_{n}$ such as, for all $\left(u_{1}, \ldots, u_{n}\right) \in$ $[0,1]^{n}$,

$$
C\left(u_{1}, \ldots, u_{n}\right)=\exp \left\{\sum_{i=1}^{n} \tilde{u}_{i} A_{C}\left(\frac{\tilde{u}_{i}}{\sum_{i=1}^{n} \tilde{u}_{i}}, \ldots, \frac{\tilde{u}_{n-1}}{\sum_{i=1}^{n} \tilde{u}_{i}}\right)\right\}, \tilde{u}_{i}=\log u_{i} .
$$

We characterize here a conditional distribution whose underlying copula belongs to Archimax class.

Proposition 7 Let $F_{t}$ be the n-dimensional function associated to an Archimax copula $C_{\varphi_{t}}$. Then, for every $x_{t}=$ $\left(x_{t, 1} ; \ldots ; x_{t, n}\right)$ and $u_{t}=\left(u_{t, 1} ; \ldots ; u_{t, n}\right) \in(\overline{\mathbb{R}})^{n}$ such as $x_{t, i} \in\left[0 ; u_{t, i}\right]$ the conditional distribution $F_{t, C}\left(x_{t} \mid u_{t}\right)$ is given, for all $t \in \chi$ and $y_{t}=F_{t}\left(x_{t}\right)$, by

$$
\text { i) } F_{t, C}\left(x_{t} \mid u_{t}\right)=S_{\varphi_{t}}\left(y_{t}, u_{t}\right) \text { for a specific function } S_{\varphi_{t}}:[0,1]^{n} \times[0,1]^{n} \longrightarrow\left[0,1\left[\text { if } C_{\varphi_{t}}\right. \text { is strictly Archimedean. }\right.
$$


ii) $F_{t, C}\left(x_{t} \mid u_{t}\right)=\exp \left\{-D_{\varphi_{t}}\left(y_{t}, u_{t}\right)\right\}$ where $D_{\varphi_{t}}$ is a conditional measure defined on $[0,1]^{n} \times[0,1]^{n}$, if $C_{\varphi_{t}}$ is strictly extremal.

Proof. Let's consider two realizations $x_{t}=\left(x_{t, 1} ; \ldots ; x_{t}, n\right) \in(\overline{\mathbb{R}})^{n}$ and $u_{t}=\left(u_{t, 1} ; \ldots ; u_{t, n}\right) \in(\overline{\mathbb{R}})^{n}$ of the process $X$ such as, $0 \leq x_{t, i} \leq u_{t, i}$. It follows that,

$$
F_{t, C}\left(x_{t} \mid u_{t}\right)=P\left(\bigcap_{i=1}^{n}\left(X_{t, i} \leq x_{t, i}\right) \mid \bigcap_{i=1}^{n}\left(X_{t, i} \leq u_{t, i}\right)\right),
$$

Moreover, note that $F_{U_{t, i}}(x)=x$ for the distribution function of standard uniform law. So, from (24) it follows that

$$
F_{t, C}\left(x_{t} \mid u_{t}\right)=P\left(\bigcap_{i=1}^{n}\left(F_{t, i}\left(X_{t, i}\right) \leq y_{t, i}\right) \bigcap_{i=1}^{n}\left(F_{t, i}\left(X_{t, i}\right) \leq u_{t, i}\right)\right) ;
$$

where, for a fixed $t \in \chi$ and $y_{t, i}=F_{t, i}^{-1}\left(x_{t, i}\right)$. Moreover, since for all $t \in \chi$, the variables $U_{t, i}$ are still the probability integral transformations, $U_{t, i} \sim F_{t, i}\left(X_{t, i}\right)$. Then from (25), it follows that

$$
F_{t, C}\left(x_{t} \mid u_{t}\right)=\frac{P\left(\bigcap_{i=1}^{n}\left(U_{t, i} \leq y_{t, i}\right) ; \bigcap_{i=1}^{n}\left(U_{t, i} \leq u_{t, i}\right)\right)}{P\left(\bigcap_{i=1}^{n}\left(U_{i} \leq u_{i}\right)\right)}=\frac{P\left(\bigcap_{i=1}^{n}\left(U_{t, 1} \leq y_{t, 1}\right)\right)}{P\left(\bigcap_{i=1}^{n}\left(U_{t, 1} \leq u_{t, 1}\right)\right)} .
$$

Furthermore, from (1) every copula $\mathrm{C}$ is the distribution function of uniform vector $\left(U_{1}, \ldots, U_{n}\right)$ even $U_{i} \sim H_{i}\left(X_{i}\right)$ regardless of the original distribution $\mathrm{H}$, it results that

$$
F_{t, C}\left(x_{t} \mid u_{t}\right)=\frac{C_{\varphi_{t}}\left(y_{t, 1} ; \ldots ; y_{t, n}\right)}{C_{\varphi_{t}}\left(u_{t, 1} ; \ldots ; u_{t, n}\right)} \text { for all } \mathrm{y}_{t} \in[0,1]^{n}, u_{t} \in(\overline{\mathbb{R}})^{n}
$$

i) If $\tilde{C}_{\varphi}$ is strictly Archimedean, then

$$
F_{t, C}\left(x_{t} \mid u_{t}\right)=\frac{\varphi_{t}\left(\varphi_{t}^{-1}\left(y_{t, i}\right)+\ldots+\varphi^{-1}\left(y_{t, i}\right)\right)}{\varphi_{t}\left(\varphi_{t}^{-1}\left(u_{t, i}\right)+\ldots+\varphi_{t}^{-1}\left(u_{t, i}\right)\right)}=S_{\varphi_{t}}\left(y_{t}, u_{t}\right) .
$$

Moreover, the function $\varphi_{t}^{-1}$ is stricly increasing from $[0,+\infty]$ to $[0,1]$. Since $x_{i} \in\left[0, u_{i}\right]$, then, $\sum_{i=1}^{n} \varphi^{-1}\left(x_{i}\right) \leq$ $\sum_{i=1}^{n} \varphi^{-1}\left(u_{i}\right)$ and finaly $S_{\varphi_{t}}\left(x_{t}, u_{t}\right)<1$ since $\varphi_{t}$ is instead decreasing.

ii) If $C_{\varphi}$ is strictly an extremal model, by setting $t_{z_{t}, i}=\frac{z_{i}}{\sum_{i=1}^{n} z_{i}}, z_{t} \in\left\{x_{t}, u_{t}\right\}$ the relation (26) gives

$$
H_{C}(x \mid u)=\exp \left\{\sum_{i=1}^{n} x_{i} A_{C_{\varphi}}\left(t_{x, 1}, \ldots t_{x, 1}\right)-\sum_{i=1}^{n} \tilde{u}_{i} A_{C_{\varphi}} A_{C_{\varphi}}\left(t_{u, 1}, \ldots t_{u, 1}\right)\right\} .
$$

Finally, it follows a convex measure $D_{\varphi}$, mapping $\left[(\mathbb{R} \cup\{ \pm \infty\})^{n}\right]^{2}$ to $[0,1]$ such as

$$
D_{\varphi}(y, u)=\sum_{i=1}^{n} y_{i} A_{C_{\varphi}}\left(t_{x, 1}, \ldots t_{x, 1}\right)-\sum_{i=1}^{n} \tilde{u}_{i} A_{C_{\varphi}} A_{C_{\varphi}}\left(t_{u, 1}, \ldots t_{u, 1}\right)
$$

which proves Theorem 7 as disserted.

Let's consider a $\varphi_{t}$-Archimedean process $\left\{X_{t}, t \in \chi\right\}$ and a conditioning subset $W_{t}$. For simplification purpose, let reduce $W_{t}$ to a single element, $W_{t}=\left\{w_{t}\right\}$. The following result provides a key property of parametric conditional Archimedean copulas.

Proposition 8 For a given conditioning set $W_{t}$, the conditional copula $C_{W_{t}}$ of $X_{t} \mid W_{i}$ where $X_{t, i} \mid W_{t} \sim F_{t, i} ; 1 \leq i \leq n$ exists and it coincides with the joint distribution function of $U_{i} \equiv F_{t, i}\left(X_{t} \mid W_{i}\right) ; 1 \leq i \leq k$ given $W_{t}$. Moreover if, for the bivariate case, the Archimax copula $C_{\varphi_{t}}$ is an extremal model then $C_{W_{t}}$ is a conditional extremal copula.

Proof. For a fixed parameter $t \in \chi$ the conditioning set $\mathrm{W}_{t}$ is such that for all $u_{t}=\left(u_{t, 1}, \ldots, u_{t, n}\right) \in(\overline{\mathbb{R}})^{n} ; u_{t, i} / w_{t} \in$ $[0,1]$. Then, Theorem 2 can also be applied for the parametric case. Therefore, the relation (4) insures that $C_{W_{t}}$ exists and it coincides with the joint distribution function of the uniform vector

$$
\left(U_{t, 1} ; \ldots ; U_{t, n}\right) \sim\left(F_{t, 1}\left(X_{t, 1} \mid W_{t}\right) ; \ldots ; F_{t, n}\left(X_{t, n} \mid W_{t}\right)\right) \text { or } F_{t}\left(x_{t, 1} ; \ldots ; x_{t, n} / w_{t}\right)=C_{\varphi_{t}}\left(F_{t, 1}\left(x_{t, 1} \mid w_{t}\right) ; \ldots ; F_{t, n}\left(x_{t, n} \mid w_{t}\right)\right) .
$$


Furthermore, note that, in bivariate case, the formula (6) can be written more simply such as

$$
C_{\varphi, A}\left(u_{1}, u_{2}\right)=\varphi^{-1}\left[\left(\varphi\left(u_{1}\right)+\varphi\left(u_{2}\right)\right) A\left(\frac{\varphi\left(u_{1}\right)}{\varphi\left(u_{1}\right)+\varphi\left(u_{2}\right)}\right)\right] ;\left(u_{1}, u_{2}\right) \in[0,1]^{2} ;
$$

Using both parametric and condtional context, (29) gives, for all $\left(u_{t}, v_{t}\right) \in[0,1]^{2}, t \in \chi$.

$$
C_{\varphi_{t}, A}\left(u_{t}, v_{t} / w_{t}\right)=\varphi_{t}^{-1}\left[\left(\varphi_{t}\left(u_{t} \mid w_{t}\right)+\varphi\left(u_{t} \mid w_{t}\right)\right) A\left(\frac{\varphi\left(u_{t} \mid w_{t}\right)}{\varphi\left(u_{t} \mid w_{t}\right)+\varphi\left(u_{t} \mid w_{t}\right)}\right)\right] .
$$

Moreover, for Archimax copulas, a copula is also extremal if its parametric generator is given by $\varphi_{t}(x)=-\ln x$ in the relation (30). So, the copula $C_{\varphi_{t}, A}$ is such as

$$
C\left(\left(u_{t, 1} \mid w_{t}\right),\left(u_{t, 2} \mid w_{t}\right)\right)=\exp \left\{\left(\ln \left(u_{t, 1} \mid w_{t}\right)+\ln \left(u_{t, 2} \mid w_{t}\right)\right) A\left(\frac{\ln \left(u_{t, 1} \mid w_{t}\right)}{\ln \left(u_{t, 1} \mid w_{t}\right)+\ln \left(u_{t, 2} \mid w_{t}\right)}\right)\right\} .
$$

By making the variable change $v_{t, i}=u_{t, i} \mid w_{t}$, we have $v_{t, i} \in[0,1], 1 \leq i \leq 2$ and (31) gives rather

$$
C\left(v_{t, 1} ; v_{t, 2}\right)=\exp \left\{\left(\bar{v}_{t, 1}+\bar{v}_{t, 2}\right) A\left(\frac{\bar{v}_{t, 1}}{\bar{v}_{t, 1}+\bar{v}_{t, 2}}\right)\right\},\left(v_{t, 1} ; v_{t, 2}\right) \in[0,1]^{2}, \tilde{v}_{t, i}=\log v_{t, i} .
$$

The formula (32) is the 2-dimensional case of (23) which characterize the extremal copulas.

\section{Conclusion and Discussion}

The results of the study show that the survival copula associated to a Archimedean copula can also be expressed analytically via the Archimedean generator of the original copula. We have also proved that the distribution function describing a uniform vector of survival distribution is a survival copula in Archimedean context. Conditional distribution and copulas have also been charcterized both for simple Archimedean field and in Archimax classes.

These are very interesting results at different levels. First characterizing analytically the survival copula provides an explicite form involving more tractability. Morover, that consist also in characterizing analytically the dual copula given by the survival distribution of the original copula. Another important thing is to have introduced the concept of Archimedean process. The specificity of our paper on the topic of stochastic processes analysis is that it investigates both survival and conditional properties of Archimedean copulas.

\section{References}

Bouyé, E. (2002). Multivariate Extremes at Work for Portfolio Risk Measurement. Warwick Business School Working Paper Series, WP01-10.

Charpentier, A., \& Segers, J. (2009). Tails of multivariate Archimedean copulas. J. Multivariate Anal., 100, 1521-1537. http://dx.doi.org/10.1016/j.jmva.2008.12.015

Cherubini, U., Luciano, E., \& Vecchiato, W. (2004). Copula Methods in Finance. Chichester: Wiley.

Clayton, D. G. (1978). A model for association in bivariate life tables and its application in epidemiological studies of familial tendency in chronic disease incidence. Biometrika, 65, 141-151.

Di Ciaccio, A., Coli, M., Ibanez, A., \& Miguel, J. (Eds.) (2012). Advanced Statistical Methods for the Analysis of Large Data-Sets, Studies in Theoretical and Applied Statistics. Heidelberg, Berlin: Springer-Verlag.

Durante, F., Härdle, W., \& Rychlik, T. (2010). Copula Theory and Its Applications, Proceedings of the Workshop Held in Warsaw 25-26, Springer.

Embrechts, P. A., McNeill, A., \& Straumann, D. (2001). In M. Dempster, \& H. K. Moatt (Eds.), Correlation and Dependence in Risk Management: Properties and Pitfalls in Risk Management: Value at Risk and Beyond. Cambridge University Press.

Frahm, G. (2006). On the extremal dependence of multivariate distributions. Stat. Probability Letters, 76, 14701481.

Genest, C., \& MacKay, R. J. (1986). Copules archimé diennes et familles de lois bidimensionelles dont les marges sont données. Canadian J. of Statistics, 14, 145-159.

Hounès, H. (1999). Représentation uniforme des lois multidimensionnelles et mesures de dépendance. 
Joe, H. (1997). Multivariate Models and Dependence Concepts. Monographs on Statistics and Applied Probabilty, 73. London: Chapman and Hall.

Klugman, S. A., \& Parsa, R. (1999). Fitting bivariate loss distributions with copulas. Insurance Math. Econom., 24, 139-148.

Larsson, M. (2011). Tail Properties of Multivariate Archimedean Copulas. Masters Thesis, ETH Zürich. Corneil University Library.

McNeil, J. A. (2008). Sampling nested Archimedean copulas. Journal of Statistical Computation and Simulation, $78(6), 567-581$.

McNeil, J. A., \& Nešlehová, J. (2009). Multivariate Archimedean copulas, D-monotone functions and $l_{1}$-norm symmetric distributions. The Annals of Statistics, 37(5B), 3059-3097. http://dx.doi.org/10.1214/07-AOS556

Müller, A., \& Scarsini, M. (2005). Archimedean copulae and positive dependence. Journal of Mulivariate Analysis, 93, 434-445.

Nelsen, R. B. (2007). An Introduction to Copulas. Springer Series in Statistics (2nd ed.). New York: Springer.

Patton, A. J. (2006). Modelling asymmetric exchange rate dependence. International Economic Review, 47(2), 527-556.

Pickands, J. (1981). Multivariate extreme values distributions-in Bulletin of Int. Statistical Institute, Procedings, 43rd Session, Buenos Aires, 1981, pp. 859-878.

Schmitz, V. (2003). Copulas and Stochastic Processes, Rheinisch-Westfälische Technische Hochschule Aachen. Retrieve from http://darwin.bth.rwth-aachen.de/opus3/volltexte/2004/935/pdf/Schmitz_Volker.pdf (2009-0225)

Sklar, A. (1959). Fonctions de repartition à n dimensions et leurs marges-Publications de l'Institut de Statistique de l'Université de Paris, 8, pp. 229-231. 